Voix et Images

voixetimages

\title{
Entre la quête et la métalittérature - Aquin et Cortázar comme représentants du postmoderne excentrique
}

\section{Amaryll Chanady}

Volume 12, numéro 1 (34), automne 1986

Québec-Amérique latine

URI : https://id.erudit.org/iderudit/200605ar

DOI : https://doi.org/10.7202/200605ar

Aller au sommaire du numéro

Éditeur(s)

Université du Québec à Montréal

ISSN

0318-9201 (imprimé)

1705-933X (numérique)

Découvrir la revue

Citer cet article

Chanady, A. (1986). Entre la quête et la métalittérature - Aquin et Cortázar comme représentants du postmoderne excentrique. Voix et Images, 12(1),

42-53. https://doi.org/10.7202/200605ar d'utilisation que vous pouvez consulter en ligne.

https://apropos.erudit.org/fr/usagers/politique-dutilisation/ 


\title{
Entre la quête et la métalittérature - Aquin et Cortázar comme représentants du postmoderne excentrique
}

\author{
par Amaryll Chanady, Université de Montréal
}

En 1963, l'écrivain argentin Julio Cortázar a publié un roman qui est rapidement devenu un best-seller, lu et discuté par un grand nombre de lecteurs en dépit de sa complexité structurelle, sa rupture avec les traditions romanesques, et ses innombrables références érudites. Il s'agit de Marelle, cette œuvre monumentale qui attaque les conventions littéraires, ainsi que les habitudes paresseuses des lecteurs femelles - un terme que Cortázar allait remplacer plus tard par lecteurs passifs - et s'interroge longuement sur son propre statut fictif et celui de la littérature en général. Marelle s'insère donc dans le cadre de la métalittérature (ou métafiction, appellation plus en usage en Amérique du nord) qui a commencé avec Don Quichotte, et s'est développée à travers l'œuvre de Lawrence Sterne et de Diderot, pour renaître avec la postmodernité du vingtième siècle, après être passée par une période relativement creuse à l'époque du réalisme et du naturalisme. L'autoconscience du narrateur et la prolifération des méta-discours rappellent l'œuvre de John Barth, de Vladimir Nabokov, de John Fowles et d'Italo Calvino, et la consacrent comme ceuvre postmoderniste par excellence. La date de publication, d'ailleurs, n'a apparemment rien d'étonnant, car c'est justement dans les années soixante que la métalittérature prend un grand essor ${ }^{1}$.

Mais comment, sans simplifier à outrance, situer Cortázar dans la lignée de Barth, Nabokov, Fowles et Calvino qui, eux, appartiennent à des cultures dotées d'une longue tradition littéraire, reconnue bien en dehors des frontières nationales? Même si Jorge Luis Borges s'est fait connaître il y a longtemps dans tout l'Occident avec les nombreuses traductions de ses récits étonnants et originaux, il reste un cas assez isolé. Jusqu'au "boom» latino-américain des années soixante, la production littéraire argentine, comme celle de toute l'Amérique du sud (exception faite de Ruben Dario connu au moins en Espagne), appartenait à une culture "excentrique». Au complexe d'infériorité qu'avaient beaucoup d'écrivains latino-américains vis-à-vis des cultures bien établies des continents nord-américain et européen, s'ajoutait une identité problématique, produite par le métissage et le choc des cultures. Cette situation donne souvent naissance à une littérature qui cherche à établir et à affirmer une identité nationale. On n'a qu'à penser au courant dit "magico-réaliste» qui voulait représenter le Sud-américain «authentique», comme le mayaquiché guatémaltèque avec sa vision magique du monde et sa croyance dans le surnaturel, qu'on trouve dans les romans de Miguel Angel Asturias. Il peut donc paraître surprenant que Cortázar, qui était fort conscient de ce problème d'identité (même si l'Argentine est beaucoup plus européenne que tous les autres pays latino-américains où il $\mathrm{y}$ a une proportion importante d'Amérindiens), puisse allier cette thématique à une réflexion métalittéraire constante, qui semble plutôt propre à la production littéraire de cultures bien ancrées dans leur identité et prêtes à interroger le statut et la forme du texte. Telle est pourtant la réussite de Cortázar. 
Deux années après la publication de Marelle, il se produit un autre phénomène littéraire important - la parution, en 1965, dans un pays situé à des milliers de kilomètres de l'Argentine, de Prochain épisode d'Hubert Aquin. L'auteur québécois a aussi réalisé un chef-d'œuvre dans lequel la recherche et l'affirmation d'une identité n'ont pas relégué au second plan une recherche formelle qui s'apparente à celle de Cortázar sous certains aspects et situe sans aucun doute Prochain épisode dans la catégorie des classiques postmodernes. S'interrogeant sans cesse sur sa forme et sa fonction, cette œuvre s'insère également dans le cadre de la métalittérature, et opère une rupture nette avec les traditions littéraires québécoises. L'importance de ce livre, ainsi que des romans aquiniens qui le suivent, semble donc analogue à celle de Marelle pour la littérature sud-américaine.

En dépit de ces similitudes apparentes, cependant, il faut se demander si on peut effectivement comparer deux écrivains si différents venant de deux cultures très éloignées l'une de l'autre. Cortázar, écrivain prolifique, lu en espagnol et en traduction dans des douzaines de pays, invité à des colloques et des réceptions partout dans le monde, et installé pendant trente-trois ans, depuis 1951 jusqu'à sa mort en 1984, à Paris, semble avoir peu en commun avec Aquin. Désillusionné, et incapable de continuer à écrire, ce dernier s'est suicidé en 1977 après quatre romans et plusieurs textes courts. Quoique son premier roman ait provoqué une forte réaction dans le milieu intellectuel québécois, et qu'on lui ait décerné plusieurs prix par la suite, Aquin se sentait profordément découragé par l'enthousiasme décroissant du public pour son œuvre, par ses dettes constantes et l'impuissance qu'il ressentait à l'égard de la situation politique et culturelle du Québec. Cortázar, au contraire, devenait de plus en plus célèbre avec chaque nouvelle publication. Quoique pour Aquin la situation s'améliore aujourd'hui, a vec les nombreuses études qui lui sont consacrées, Cortázar a toujours joui d'une meilleure reconnaissance internationale.

On peut aussi se demander en quoi la situation culturelle du Québec, avec ses six millions d'habitants entourés par deux cent cinquante millions d'anglophones, ressemble à celle de l'Argentine qui a cinq fois plus d'habitants, et fait partie d'un continent de plus de trois cents millions d'hispanophones. Si le français est minoritaire en Amérique du nord, l'espagnol, par contre, est répandu dans presque tout le continent sud-américain. Il est vrai que du point de vue ethnique, la population du Québec est plus homogène que celle de l'Argentine, dont plus de $50 \mathrm{p}$. cent de ses hispanophones sont de descendance italienne. À propos du reste de l'Amérique dite latine, Javier Garcia Méndez parle dans un article récent de l'hétérogénéité qui est l'essence même de cette Amérique non pas latine mais métisse ${ }^{2}$. Une différence d'attitude entre les deux cultures est aussi suggérée, d'un côté, par la déclaration faite par Cortázar en 1982, selon laquelle les écrivains latino-américains se sentent offensés de n'être pas suffisamment connus à l'étranger ${ }^{3}$; et de l'autre, par l'évocation que faisait Aquin dans "la Fatigue culturelle du Canada français» (1962) de la prise de conscience minoritaire: l'autopunition, le masochisme, l'autodévaluation ${ }^{4}$. 
En dépit de ces différences, pourtant, on peut rapprocher l'œuvre de ces deux écrivains pour deux raisons principales. L'une, mentionnée plus haut, est l'aspect métalittéraire qui la caractérise et l'originalité de Marelle et de Prochain épisode par rapport à la production littéraire de l'époque dans chacune des deux aires culturelles. L'autre est la présence de plusieurs thèmes, reliés à des problèmes d'identité, qui se manifestent constamment chez Aquin et Cortázar, et qu'une étude comparatiste aidera à mieux situer.

Le premier de ces thèmes, la fascination pour l'Europe, a pu être critiquée chez Aquin. Jean-Guy Rens, par exemple, dans un article très négatif intitulé "Un double échec littéraire... et politique», condamne l'auteur québécois non seulement pour le maniérisme et la préciosité, par lesquels il prétendrait impressionner le lecteur ${ }^{5}$, mais aussi pour son admiration pour l'Europe, évidente dans tous ses romans. Le héros de Prochain épisode contemple avec fascination les objets d'art dans le château d'Echandens. Ils lui font oublier momentanément son projet meurtrier: Ah, vraiment je veux vivre ici, dans cette retraite empreinte de douceur et où s'exprime un vouloir-vivre antique qui ne s'est pas perdu ${ }^{6}$. Le raffinement et l'élégance du style de vie de $\mathrm{H}$. de Heutz provoquent chez le héros un sentiment d'infériorité. Is suscitent d'ailleurs en lui une grande admiration pour l'homme mystérieux qui hante élégamment (PE, p. 106) son château. La narratrice de l'Antiphonaire ${ }^{7}$, Christine Forestier, prépare sa thèse de doctorat sur Jules-César Beausang, un médecin érudit du XVI ${ }^{e}$ siècle, et passe de longues heures à rêver aux penseurs européens de la Renaissance, s’identifiant même à des personnes qui ont été en contact avec le manuscrit du médecin suisse. Le jeune couple dans Neige noire ${ }^{8}$ s'extasie devant la beauté de la Norvège, qui représente le lieu d'une quête, d'une transformation personnelle, et permet encore davantage à Nicolas de s'identifier à Fortinbras, le héros légendaire dont il a joué le rôle au théâtre ${ }^{9}$. Dans Trou de mémoire ${ }^{10}$, le tableau des deux ambassadeurs par Holbein le Jeune a une importance capitale dans la structure et la signification du roman, où une grande partie de l'intrigue se situe également en Europe.

Aquin lui-même explique son admiration pour l'Europe par la pauvreté culturelle qu'il voit dans son propre pays: Comment évaluer le capital d'une société, le capital humain, culturel? Et comme il y en a très peu ici, alors j'ai cherché ailleurs, [...] le Québec n'a que trois cents ans d'histoire et c'est pauvre $[\ldots]^{11}$. Mais Aquin reconnaît que se tourner vers l'Europe ne résoud rien. Il déplore le déracinement du Canadien français qui doit s'exiler en France pour réussir, qui s'abolit dans l'excentricité', refuse son centre de gravité, cherche désespérément ailleurs un centre et erre dans tous les labyrinthes qui s'offrent à lui ${ }^{12}$. Celui-ci est donc deux fois apatride ${ }^{13}$.

Dans le cas de Cortázar, la littérature et la culture européennes ont également eu une grande influence. Mais pour l'auteur argentin, l'attrait de l'Europe était si fort qu'il y a passé trente-trois ans de sa vie, même s'il continuait à écrire en espagnol. Beaucoup d'Argentins, en effet, rêvaient à Paris à l'époque, car la capitale française représentait pour eux le centre de la culture et de la connaissance. Cortázar racontait que pour eux, Buenos Aires était un châtiment; $y$ vivre signifiait être emprisonné ${ }^{14}$. À dix-huit ans, il a 
même essayé de se réfugier sur un bateau pour s'enfuir en Europe avec un groupe d'amis. Les Argentins qui éprouvaient de la nostalgie pour l'Europe et ne songeaient qu'à faire un pèlerinage à Paris se sentaient comme des étrangers dans leur propre pays, des exilés en Amérique ${ }^{15}$. Comme l'expliquait l'auteur argentin H.A. Murena, l'Amérique est constituée d'exilés qui ne peuvent jamais oublier leur situation de personnes déplacées: [...] dans le passé, nous vivions dans une terre fécondée par l'esprit, qui s'appelle l'Europe, et tout d'un coup on nous a expulsés d'elle; nous sommes tombés dans une autre terre, dans une terre sans culture, vide d'esprit $[\ldots]^{16}$. Même si cette attitude caractérisait aussi d'autres Sud-Américains, c'était surtout les Argentins qu'on critiquait parce qu'ils vivaient plus que les autres dos à l'Amérique latine.

Dans Marelle, où l'Argentin se sent exilé dans son propre pays, la fascination pour Paris est l'un des thèmes les plus importants du livre. Pour le protagoniste Horacio Oliveira, Paris représente le ciel, tandis que Buenos Aires signifie l'enfer - jusqu'au moment où il arrive à Paris et se rend compte que le Paris idéalisé qu'il s'était imaginé n'existe pas. Il y est profondément conscient de sa condition d'étranger; il a l'impression de se déplacer comme une feuille sèche ${ }^{17}$ et voit que Paris ne lui permettra pas de réaliser sa quête d'un «centre». Désillusionné, il rentre enfin en Argentine. La deuxième partie du roman débute alors par une citation d'Apollinaire: Il faut voyager loin en aimant sa maison.

Dans 62/maquette à monter $(1968)^{18}$, Paris ne perd jamais son caractère magique. Le protagoniste Juan la place dans la catégorie de la "Cité", espace mystérieux qui offre la possibilité d'entrevoir le kibboutz du désir à travers les interstices (agugeros, resquicios) de la réalité, pendant les moments d'illumination qui lui permettent de saisir cette unité fulgurante ${ }^{19}$. Les autres villes qui ont ce statut privilégié sont Vienne et Londres.

Cette fascination pour l'Europe est souvent accompagnée d'un deuxième thème, celui d'un manque d'identité nationale. Pour conjurer ce vide, explique Oliveira dans Marelle, les Argentins se livrent à une grande parodie culturelle, qui consiste en criollicentrisme, banlieucentrisme, et une valorisation exagérée du folklore ( $\mathrm{M}, \mathrm{ch} .3)$. Ils se forgent une identité artificielle qui cache la véritable identité nationale. Les personnages de 62/maquette à monter se donnent un air faussement argentin avec leurs costumes rayés et cintrés, aussi ridicules à Londres qu'à Paris. Même s'ils n'assumaient pas une identité artificielle, leur désir de paraître argentins à l'étranger brimerait leur individualité. Comme le rappelle Cortázar au tout début de Marelle avec la citation de Jacques Vaché, Rien ne vous tue un homme comme d'être obligé de représenter un pays. Ce rejet d'une fausse identité nationale était à la base d'une querelle acerbe entre Cortázar et le Péruvien José Maria Arguedas, qui essayait dans ses romans et nouvelles d'exprimer la vision du monde qu'avaient les Indiens de son pays en développant un style caractérisé par un mélange de phrases espagnoles, de mots quechua, et d'une syntaxe simplifiée, pour recréer la façon de parler des indigènes. 
Ne voit-on pas la même attitude chez Aquin quand il dénonce l'utilisation du joual? Mais je ne veux pas écrire le joual, nous dit-il, car c'est se condamner d'avance à écrire pour rien, pour personne ${ }^{20}$. Aquin admet la contradiction entre la thématique de ses livres et son refus d'en assumer les particularités $d u$ dialecte $^{21}$. Quant au régionalisme, il est aussi sévère dans sa critique que Cortázar: les régionalistes, selon Aquin, ont utilisé une 'authenticité folklorique' et se sont crus ainsi plus canadiens parce qu'ils monnayaient leur enracinement à rabais [...]. Ce n'est pas en renchérissant un texte de quelques 'fleurs de terroir'... qu'un auteur peut s'acquitter de son origine ${ }^{22}$. Cette sorte de littérature ne peut produire que des "stéréotypes superficiels» et une distanciation exotique du pays natal ${ }^{23}$. Par ailleurs, il critique les auteurs qui veulent atteindre à l'universel parce que ceux-ci n'assument pas leur identité. La fausseté du stéréotype national est bien mise en évidence dans Prochain épisode, où l'image romantique de l'armée de frères québécois et celle de la femme-pays sont questionnées par l'identité ambiguë de K. La réalité, comme le suggère le roman, est bien loin de cette idéalisation.

Le manque d'identité nationale, que Cortázar mentionne déjà dans son premier roman, les Gagnants (1960), où il considère l'Argentine comme une terre de personne ${ }^{24}$, est explicitement abordé dans l'œuvre d'Aquin. Dans Trou de mémoire, ce manque est relié en partie au métissage de P.X. Magnant: Je suis d'ascendance bulgare, deuxième génération, côté maternel, avec une goutte de sang cri [...]. L'homme tribal, coureur de bois, s'est fait assimiler par l'homo bulgarus qui, à son tour, s'est fait assimiler par de vulgaires Canadiens français (TM, p. 37). Mais la cause principale de la mort spirituelle du Québec ( Notre pays est un cadavre encombrant, TM, p. 48) est la conquête par les Anglais, qui a produit une amnésie culturelle ${ }^{25}$ :

Le Québec, c'est cette poignée de comédiens bègues et amnésiques qui se regardent et s'interrogent du regard et qui semblent hantés par la platitude comme Hamlet par le spectre. Ils ne reconnaissent même pas le lieu dramatique et sont incapables de se rappeler le premier mot de la première ligne du drame visqueux qui, faute de commencer, ne finira jamais.

(TM, p. 56)

Le narrateur de Prochain épisode parle du cachot national (PE, p. 24), et de la vomissure décantée de notre histoire nationale (PE, p. 57); mais il évoque surtout son peuple inédit (PE, p. 16) qui est sans histoire (PE, p. 77).

La dimension politique de l'œuvre d'Aquin est beaucoup plus virulente que dans le cas de Cortázar; tandis que celui-là se révolte contre la situation des Québécois entourés et dominés par les Canadiens anglais, l'auteur argentin ne semble pas devoir se défendre contre une domination étrangère. Dans son roman le plus politisé, le Livre de Manuel (1973), il critique principalement les dictatures latino-américaines et la politique étrangère des États-Unis. Mais la différence est peut-être moins grande qu'il n'y paraît, comme le suggèrent ces paroles de Cortázar: 


\begin{abstract}
La littérature est pour nous une des meilleures armes en cette bataille contre ce que certains nomment encore le rêve nordaméricain, et qu'il vaudrait mieux qualifier de cauchemar nordaméricain; contre les tentatives d'asservissement culturel à base de propagande et de déculturation... ${ }^{26}$
\end{abstract}

Dans ses écrits fictifs et non-fictifs, il s'érige contre l'influence des façons de penser occidentales et rationnelles, et cherche même d'autres formes de conceptualisation de la réalité dans le bouddhisme Zen. D'autres auteurs sud-américains ont décrit la destruction de leur identité par l'acceptation de valeurs superficielles venant de l'Amérique anglophone. On n'a qu'à penser à certains romans du Mexicain Carlos Fuentes ou de l'Argentin Manuel Puig.

Étroitement lié aux deux thèmes qu'on vient de discuter et souvent inséparable de ceux-ci, est le thème de la dissolution de l'identité individuelle. Chez Cortãzar, cela se manifeste surtout par les doubles. Dans «la Lointaine» (1954), par exemple, la protagoniste Alina Reyes voit sa petite existence confortable à Buenos Aires envahie tout d'un coup par la conviction que son double mène une vie de pauvreté et de souffrance à Budapest. Incapable de continuer à participer à la vie mondaine, elle fait un voyage à la capitale hongroise, où son corps et son existence sont échangés contre ceux de la clocharde. Le jeune protagoniste de "la Nuit face au ciel» (1964) est amené à l'hôpital après un accident de motocyclette, après lequel il se convertit (ou se réincarne rétrospectivement) en une victime sacrificielle des Aztèques. Dans «l'Île à midi» (1966), un agent de bord d'une compagnie aérienne mène une vie idyllique dans une île grecque pendant qu'il continue à remplir ses fonctions à bord de l'avion. À part ces exemples de disjonction de la personnalité par la présence d'un double, les problèmes d'identité se manifestent de façon extrême et surprenante dans "Axolotl» (1964), où un visiteur au Jardin des Plantes à Paris est fasciné à tel point par les axolotl qu'il se convertit lui-même en cette espèce de larve et regarde l'homme qu'il était avant de s'éloigner de l'aquarium.

Les personnages d'Aquin ne se dédoublent pas de façon surnaturelle, mais ils s'identifient souvent à d'autres personnages. Lors de son séjour en prison, le narrateur de Prochain épisode écrit un roman d'espionnage dans lequel il investit au début son délégué de pouvoir (PE, p. 35) avec tous les attributs qu'il aimerait posséder lui-même. Le narrateur-protagoniste s'identifie ensuite à Ferragus, un personnage de Balzac: L'ombre du grand Ferragus m'abritait, son sang répandait dans mes veines une substance inflammable (PE, p. 40); non seulement veut-il assumer une identité fictive, mais celui qu'il aimerait imiter peut conjurer toute capture par ses mimétismes, ses dédoublements et ses déplacements continuels (PE, p. 8). Quand le protagoniste invente une histoire pour cacher son identité à de Heutz, il finit par s'identifier sans le vouloir au père pitoyable de sa fiction: $A$ vouloir me faire passer pour un autre, je deviens cet autre ( $\mathrm{PE}, \mathrm{p} .48)$. Mais c'est finalement à son ennemi qu'il s'identifie quand il contemple avec admiration son élégant château: [...] secrètement je suis entré en lui (PE, p. 122); à ce moment, il voit de Heutz comme le vrai double de Ferragus (PE, p. 121). La contradic- 
tion qui caractérise son identité, cependant, exige sa poursuite meurtrière de l'autre lui-même pour donner un sens à sa vie. S'il n'arrive pas, se dit-il pendant son attente au château, que vais-je devenir? (PE, p. 115).

$\mathrm{Au}$ fur et à mesure que son récit avance, le narrateur voit son identité se désagréger progressivement: Tout s'effrite au passé (PE, p. 4); j'ai peur de me réveiller dégénéré, complètement désidentifié, anéanti. Un autre que moi, les yeux hagards et le cerveau purgé de toute antériorité, franchira la grille le jour de ma libération (PE, p. 35); je n'aperçois qu'une masse protozoaire et gélatineuse qui m'épuise et me ressemble (PE, p. 55). Quand il ne retrouve plus la femme qu'il aime après son échec, il perd même le sens d'appartenance à sa patrie: Je n'ai plus de pays, on m'a oublié (PE, p. 128). D'ailleurs, l'identité du narrateur-protagoniste semble vaciller pendant tout le roman entre son aspect de révolutionnaire, celui de l'artiste incapable d'agir, et celui d'un grand déprimé27. Dans une entrevue, Aquin parle d'une désidentification et d'une désintégration totale du personnage-narrateur qui pourrait mener à une découverte d'identité28.

La "désintégration" atteint aussi P.X. Magnant dans Trou de mémoire: Le monde - qui me confère ma réalité - menace sans cesse d'éclater ( $\mathbf{P E}$, p. 69). Quand il pense au meurtre de Joan, sa réaction est analogue à celle du protagoniste de Prochain épisode: Car je t'ai perdue, mon amour, je t'ai perdue et je me perds de plus en plus moi-même [.. ] (TM, p. 98). Dans une note infra-paginale, l'éditeur fait des commentaires sur la désolidarisation de Magnant (TM, p. 112).

Christine Forestier dans l'Antiphonaire souffre d'une désintégration progressive: elle est consciente de sa vie ratée $(A, p .19)$ et accablée par la pensée qu'elle a vertement gaspillé sa jeunesse (A, p. 45). Horrifiée par son apparence physique après avoir été battue par son mari, elle est convaincue qu'elle n'a plus d'avenir: le monde s'était écroulé avec mes charmes faciaux de jeune épouse. J'étais désespérée, désenchantée de la vie, découragée... Oui, le suicide: j'y ai pensé... (A, p. 48). Elle se considère comme une sorte de gâchis vivant, un tas de pourriture... (A, p. 196), voit sa vie comme une série de séquences brisées, auto-suffisantes, dont l'addition n'égale jamais la totalité (A, p. 218), et croit qu'elle n'atteindra jamais la plénitude.

Pour conjurer le vide ou la disjonction de leur identité nationale ou personnelle, les personnages aquiniens et cortazariens poursuivent sans cesse une quête. Le narrateur-protagoniste de Prochain épisode poursuit en vain la femme-pays. P. X. Magnant, ayant été incapable de posséder totalement la femme qu'il aime (Je brûle, j'encercle, je frôle, je ne possède jamais, (TM, p. 26), essaye de trouver une "drogue nouvelle» qui lui permettrait de sortir de sa dépression: Je te cherche, mon frère, ma sceur blonde, mon amour, car j'ai soif d'aimer et d'être aimé; j'ai soif de vivre et de me mouvoir dangereusement hors du cercle mort de ma tristesse. Mais comment faire [...] (TM, p. 72). La quête est largement reliée dans ces deux romans à la situation du Québec, mais dans l'Antiphonaire et dans Neige Noire, comme le fait remarquer Jacques Pelletier, la question nationale est relayée par une nouvelle 
problématique, dont le thème central est la recherche de valeurs authentiques, du sens à donner à l'existence ${ }^{29}$. L'identification de Christine Forestier à Renata et à Antonella, ses études passionnées sur les penseurs de la Renaissance, et ses interminables rêveries qui la projettent hors de son monde (un monde qui ne lui rappelle que l'abandon de sa carrière, son mari malade et violent, et la cruauté de son amant jaloux, toutes ces activités d'évasion constituent des tentatives pour construire une vie moins vide et déprimante. Dans Neige noire, le voyage au nord de la Norvège symbolise une quête de soi-même, et au niveau plus concret, il signifie la recherche d'un nouveau départ dans la vie du couple.

Dans l'œuvre de Cortázar, la quête est souvent explicitement thématisée. Ses personnages cherchent à percer le mur du quotidien, de la réalité conventionnelle et des habitudes, pour trouver ce qui est caché derrière, un monde à jamais voilé à ceux incapables de se défaire de leurs préjugés rationnels. Le saxophoniste noir de la nouvelle «le Poursuivant» (1959) se sert de la musique, qu'il considère comme métaphysique, pour entrevoir cette réalité autre qui se dérobe sans cesse à lui: Personne ne sait ce que cherche Johnny... dans la marihuana, dans ses disques absurdes sur tant de choses, dans ses rechutes, dans le petit livre de Dylan Thomas $[\ldots]^{30}$. Mais il n'arrive jamais au but de sa quête; la porte s'ouvrant sur le monde plus «vrai» et plus authentique qu'il poursuit lui reste toujours fermée. Dans 62 /maquette à monter, Juan cherche cette réalité autre dans les "figures" ou "constellations" d'événements et de personnes éloignés dans le temps et l'espace. Les voyageurs dans les Gagnants (1960) essayent d'atteindre la poupe du bateau dans une quête qui symbolise la recherche de soi-même. Mais c'est dans Marelle que la quête est développée le plus longuement. Oliveira cherche ce qu'il appelle le kibboutz du désir, le nirvana ou le ciel de la marelle dans ses promenades à Paris, l'étude des philosophies orientales, des comportements non-conventionnels comme sa rencontre avec une vieille clocharde, ou sa relation avec la Maga qui, ayant trouvé cette autre dimension de la réalité, reste toujours inaccessible à Oliveira. De retour à Buenos Aires, il continue sa quête dans un asile, où il veut se suicider en sautant par sa fenêtre pour atterrir dans la case de la marelle qui représente le ciel.

Ce bref examen des thèmes reliés à l'identité nationale et personnelle démontre que l'œuvre de Cortâzar et d'Aquin manifeste des préoccupations qui vont bien au-delà de la recherche esthétique. Cortázar souligne, en effet, que la littérature sud-américaine

est aujourd'hui le miroir le plus net et fidèle de la rude et longue lutte que livrent peuples latino-américains pour s'approfondir en leur identité, pour découvrir leurs authentiques racines, afin de mieux appuyer leurs pieds sur la terre au moment de produire ce saut en avant qu'est la conquête ou la reconquête de leur souveraineté, de leur autodétermination ${ }^{31}$.

Il y a des parallèles évidents entre cette perspective et celle de Magnant, qui écrit au niveau de pur blasphème (TM, p. 57) pour éveiller la lucidité de son peuple, une lucidité qui agirait sans doute comme un facteur de détoxication 
et débalancerait ce charmant équilibre où tout le poids graisseux du conquérant écrase, avec des raffinements de Chinois, le corps famélique et déboitté de celui qui attend de ressusciter [...] (TM, p. 39).

Mais écrire seulement au niveau de pur blasphème n'atteindrait pas le but que se proposait Aquin. Ce dont il s'est rendu compte, c'est qu'il faut changer les habitudes de lecture, et éviter la possibilité de consommer la littérature de façon passive. Dans «la Disparition élocutoire du poète (Mallarmé)", Aquin considère le rapport écriture/lecture comme "constitutif du phénomène littéraire»: Ainsi, la littérature existe pleinement non pas quand l'œuvre est écrite, mais quand un lecteur remonte le cours des phrases et des mots pour devenir, par ce moyen, cocréateur de l'auvre ${ }^{32}$. Cette insistance sur la participation active du lecteur qui caractérise grand nombre d'œuvres post-modernes (voir Si par une nuit d'hiver un voyageur (1978) d'Italo Calvino, ou Lost in the Funhouse (1968) de John Barth, par exemple) est admirablement actualisée dans Trou de mémoire. Dans une entrevue réalisée en 1974, Aquin expliquait qu'il essayait d'étreindre le lecteur littéralement dans Trou de mémoire, ou de le violer même, à la limite; il parlait d'opération dirigée vers le lecteur étant donné que c'est lui qui crée le livre ${ }^{33}$. Chaque narrateur dans ce roman lit les textes des autres (Magnant, RR, l'éditeur, Ghezzo-Quénum), et critique, nie, corrige ou interprète les lignes soumises à ses commentaires métatextuels. Chaque narrateur discute aussi de son propre texte et sa situation d'énonciation.

Les aspects métalittéraires de ce roman, dont la discussion par $\mathrm{R} R$ de la mise en abyme que constitue le tableau de Holbein le Jeune par rapport à la macro-structure du récit, sont trop complexes pour être examinés en détail dans une si brève étude ${ }^{34}$. Ils caractérisent aussi, mais de façon moins complexe, les autres romans d'Aquin. Le narrateur de Prochain épisode parle d'emblée de son projet d'écriture, écrire un roman d'espionnage (PE, p. 1), et de son héros qu'il ne survalorisera pas: ni Sphynx, ni Tarzan extra-lucide, ni Dieu, ni Saint-Pierre... (PE, p. 2). Il fait des commentaires sur son écriture hautement automatique qui lui évite la lucidité homicide (PE, p. 6): Je farcis la page de hachis mental, j'en mets à faire craquer la syntaxe, je mitraille le papier nu... (PE, p. 6). Plus loin il admet qu'il s'introduit lui-même dans son roman (PE, p. 11) et qu'il n'invente jamais ( $\mathrm{PE}, \mathrm{p} .74)$; il dénonce même la vanité fondamentale de l'entreprise d'originalité (PE, p. 74). Il compare la forme de son récit avec celle de sa propre vie: La seule forme que je poursuis confusément depuis le début du récit, c'est la forme informe qu'a prise mon existence emprisonnée... (PE, p. 76). Les commentaires métalittéraires du narrateur portent sur la situation d'énonciation, le style du récit (autoreprésentation du code, comme le dirait Janet Paterson) ${ }^{35}$, et l'univers des personnages. Même si cela ressemble à d'autres romans sur le roman, Prochain épisode reste profondément original, à cause de la fusion graduelle entre le narrateur et son personnage ${ }^{36}$.

Christine Forestier discute aussi sa situation d'énonciation: je compose ce livre calmement, froidement, sans hallucinogène pour me stimuler... (A, p. 18); j'ai commencé ce livre sans raison sur la plage de Santa Barbara, sous 
forme de récit (A, p. 44). Elle fait des commentaires sur son style, parfois indirectement: ainsi, quand elle qualifie les livres de Beausang comme un torrent d'épithètes et un abus des procédés littéraires énigmatiques, et parle de son penchant manifeste pour le grandiose, l'énigme et le cacozélie (A, p. 109), elle vise son propre récit. C'est aussi le cas quand elle explique que Chigi avait l'intention d'intégrer son propre journal intime... au texte du célébre médecin gantois (A, p. 175). Elle analyse également la structure de son roman, qui se déplie dans un thème, et dans une reprise du thème (introductio), après quoi la 'partitio' se déroule en plusieurs points... après quoi elle confirme, pérore, argumente, déclare, divise; et établi $[t]$ des correspondances entre les diverses parties de $[s]$ on discours (A, p. 204).

Le scénario de film qui constitue la plus grande partie de Neige noire est constamment soumis à des commentaires métafictionnels. Il y a des discussions générales sur l'art filmique: Peu de films contiennent des paliers de répit, des temps vraiment morts... (NN, p. 114); le film, mieux que tout autre moyen d'expression, rend bien cette fluidité achérontique, dans la mesure au moins où il ne s'arrête jamais avant la fin de sa propre projection (NN, p. 157). Un des personnages résume même la trame du roman: Le film raconte l'histoire d'un comédien qui quitte son métier pour faire un film qui ne sera que le compte rendu de sa vie... C'est comme un film dans un film (NN, p. 155). Le narrateur offre également des interprétations de son scénario, telle l'explication que la petite valise de Nicolas est le cercueil symbolique de Sylvie (NN, p. 127). Il discute aussi de la probable réception du film l'apparition d'Éva Vos qui déconcerte le spectateur (NN, p. 56) - et du comportement du spectateur qui se trouve dans une position faetale, porté par le film qu'il regarde (NN, p. 70).

Cette autoconscience du narrateur oblige le lecteur à réfléchir à la forme et à la signification du roman, et empêche toute identification naïve aux personnages. En plus, la forme complexe des romans d'Aquin nécessite une lecture - ou plutôt des lectures - actives, ce qui contribue au projet aquinien d'amener le lecteur à être «cocréateur de l'œuvre». Ce projet, loin d'être une tentative de se complaire dans la gratuité de la recherche esthétique exagérée pour mystifier le lecteur, est profondément lié au désir qu'avait Aquin de forcer le lecteur à réfléchir aux problèmes extralittéraires abordés par ses romans.

Comme Aquin, Cortázar condamne le lecteur passif qui consomme simplement une œuvre littéraire. Ce lecteur, qu'il appelle un lecteur femelle $(M$, ch. 79), lit les romans du début à la fin comme un enfant sage ( $M$, ch. 99). Cortázar, par contre, veut provoquer le lecteur en brisant toutes les conventions, ce qui va l'obliger à devenir un "complice» (M, ch. 79) de l'auteur: $I l$ ne s'agit pas de remplacer la syntaxe par l'écriture automatique ou n'importe quel autre truc à la mode. Ce qu'il veut, c'est de transgresser le fait littéraire total, le livre, si vous voulez... (M, ch. 99). Les nombreuses discussions métalittéraires de Morelli, qui s'appliquent souvent au texte de Marelle, amènent le lecteur à s'interroger sur le statut et la forme de la littérature, et sur toutes les conventions et habitudes de sa propre vie. Le métatexte n'est 
pourtant qu'une façon de rendre le lecteur plus actif: l'autre est la forme même de Marelle, avec son mode d'emploi qui oblige le lecteur à sauter du chapitre 73 au chapitre premier, ensuite du deuxième au chapitre 116, et ainsi de suite, ce qui empêche une lecture linéaire et commode de la trame, sans cesse interrompue par les chapitres métalittéraires. Dans le chapitre 62 , le narrateur esquisse le projet d'un roman où le comportement des personnages serait inexplicable: les acteurs paraîtraient fous ou totalement idiots [...]. Tout serait comme une inquiétude, une agitation, un déracinement continu, un territoire où la causalité psychologique cèderait, déconcertée [...]. Cortázar réalise en effet ce projet dans 62 /maquette à monter, oủ les personnages se promènent sans but apparent dans trois capitales européennes, et le lecteur doit chercher le sens de l'œuvre en reconstruisant les «figures" ou "constellations" formées par les éléments du récit pour trouver lui-même les «interstices" qui laissent entrevoir une réalité autre que la conventionnelle.

Chez Aquin et chez Cortázar, la quête dans toutes ses formes ne conduit pas à une littérature naïvement engagée, insouciante de l'élaboration formelle; par ailleurs, la métalittérature et la recherche esthétique n'aboutissent pas à une complexité gratuite et aride. Les deux auteurs ne tombent ni dans le piège du didactisme, ni dans celui de la préciosité, et restent en même temps des fabulateurs accomplis, dont les œuvres figurent parmi les grandes réussites de la littérature postmoderne.

1. Voir à ce sujet Linda Hutcheon, Narcissistic Narrative: the Metafictional Paradox, Waterloo, Wilfrid Laurier University Press, 1980, p. I.

2. Javier Garcia Méndez, "Roman et science en Amérique latine,», Nuit blanche, no 23, Mai/juin 1986, p. 47.

3. Julio Cortázar, "Paroles inaugurales", prononcées lors de la Réunion d'intellectuels nord-américains et latino-américains à Mexico en septembre 1982, et publiées dans Liberté, no 148, août 1983, p. 8.

4. Liberté, vol. 4, no 23, mai 1962, p. 299-325.

5. Jean-Guy Rens. "Un double échec littéraire... et politique», le Québec littéraire, no 2, 1976, p. 121.

6. Prochain épisode, Montréal, Éditions du Renouveau pédagogique, 1969, p. 105. Toutes les références sont à cette édition et seront indiquées entre parenthèses dans le texte après l'abréviation PE.

7. L'Antiphonaire, Montréal, Le Cercle du Livre de France, 1969.' Toutes les références sont à cette édition et seront indiquées entre parenthèses dans le texte après l'abréviation A.

8. Neige noire, Montréal, Pierre Tisseyre, 1978. Toutes les références sont à cette édition et seront indiquées entre parenthèses dans le texte après l'abréviation NN.

9. Voir l'article de Françoise Maccabée-Eqbal intitulé: «l'Appel du Nord dans Neige noire: la quête de Narcisse", Voix et images, vol. V, no 2, hiver 1980, p. 365-377.

10. Trou de mémoire, Montréal, Le Cercle du Livre de France, 1968. 'Toutes les références sont à cette édition et seront indiquées entre parenthèses dans le texte après l'abréviation TM.

11. "Hubert Aquin et le jeu de l'écriture", entrevue réalisée par Anne Gagnon en 1973, et publiée dans Voix et images, vol. 1, no 1, septembre 1975, p. 5-18.

12. "La Fatigue culturelle du Canada français", repris dans Blocs erratiques, Montréal, les Quinze, 1982, p. 96. 
13. Ibid., p. 94 .

14. Luis Harss, «Julio Cortázar o la cachetada metafisica», in Los Nuestros, Buenos Aires, Sudamericana, 1977, p. 257. La traduction est la nôtre.

15. Voir Fernando Ainsa, «Las dos orillas de Julio Cortázar ", in Julio Cortázar, dir. par Pedro Lastra, Madrid, Taurus, 1981, p. 35.

16. H.A. Murena, El Pecado original de America, Buenos Aires, Sur, 1954, p. 24. La traduction est la nôtre.

17. Marelle, Ch. 3. Toutes les citations seront prises dans l'édition espagnole de Rayuela, Madrid, Catedra, 1984, et traduites par nous. Les numéros des chapitres seront indiqués dans le texte entre parenthèses, après l'abréviation $M$.

18. 62/modelo para armar, Barcelona, Bruguera, 1982.

19. Ibid., p. 10.

20. «Hubert Aquin et le jeu de l'écriture», op. cit., p. 10.

21. Ibid., p. 10 .

22. "La Fatigue culturelle du Canada français", op. cit., p. 95-96.

23. Ibid., p. 96.

24. "Tierra de nadie". Los premios, Buenos Aires, Sudamericana, 1960, p. 305.

25. Voir Patricia Smart, Hubert Aquin, agent double, Montréal, Presses de l'Université de Montréal, 1973, p. 22, et Albert Memmi, Portrait du colonisé, Paris, Jean-Jacques Pauvert, 1966, p. 139-140.

26. "Paroles inaugurales", op. cit., p. 7 .

27. Ces trois aspects correspondent, comme le souligne Patricia Smart, aux trois identités possibles de H. de Heutz, op. cit., p. 52.

28. "Hubert Aquin et le jeu de l'écriture". op. cit.. p. 14

29. Jacques Pelletier, "Sur Neige noire: l'œuvre ouverte de Hubert Aquin", Voix et images, vol. 1, no 1, septembre 1975, p. 21.

30. "El perseguidor", in El perseguidor y otros relatos, Barcelona, Bruguera, 1979, p. 325. La traduction est la nôtre.

31. "Paroles inaugurales", op. cit., p. 7.

32. Blocs erratiques, op. cit., p. 264-266.

33. "Aquin par Aquin", le Québec littéraire, no 2, 1976, p. 134.

34. Voir surtout l'analyse détaillée qu'en a faite Patricia Smart dans Hubert Aquin, agent double, op. cit., p. 89-98, celle de Sylvia Söderlind dans «Hubert Aquin et le mystère de l'anamorphose", Voix et images, vol. IX, no 3, printemps 1984, p. 103-1ll et celle de Pierrette Malcuzynski, "Anamorphose, perception carnavalisante et modalités polyphoniques dans Trou de mémoire", Voix et images, vol. XI, no 3, printemps 1986, p. 475-494.

35. Janet Paterson, «l'Autoreprésentation: formes et discours", Texte, no 1, p. 177-194.

36. Voir Agnes Whitfield, «la Problématique de la narration dans le roman québécois écrit à la première personne depuis 1960 ", thèse de doctorat, Université Laval, 1981, p. 404-414.

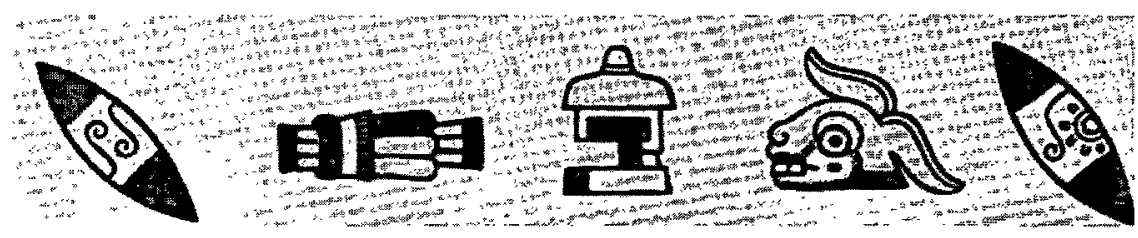




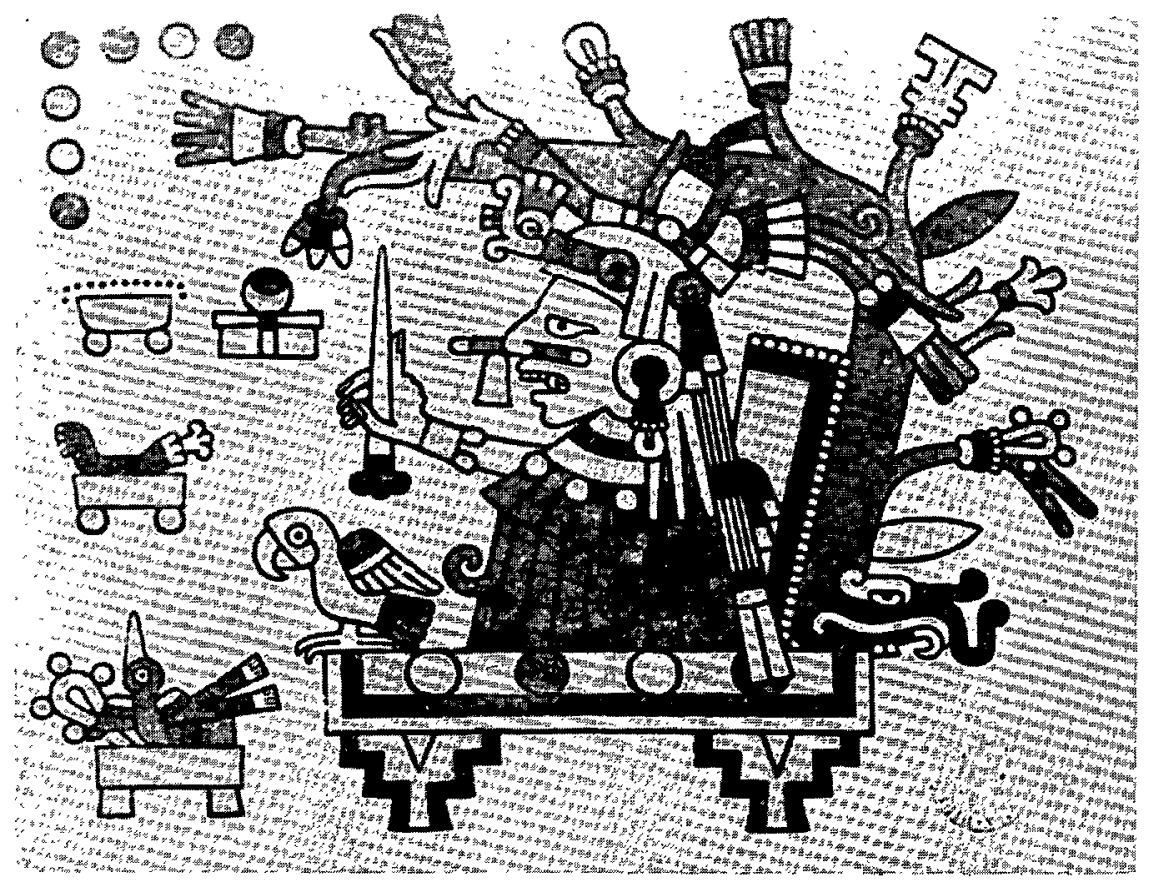

